\title{
Coupling Thermal Atomic Vapor to Slot Waveguides
}

\author{
Ralf Ritter, ${ }^{1}$ Nico Gruhler, ${ }^{2,3}$ Helge Dobbertin, ${ }^{4}$ Harald Kübler, ${ }^{1}$ Stefan Scheel, ${ }^{4}$ \\ Wolfram Pernice, ${ }^{2,3}$ Tilman Pfau, ${ }^{1}$ and Robert Löw, ${ }^{1, *}$ \\ ${ }^{1}$ 5. Physikalisches Institut and Center for Integrated Quantum Science and Technology, \\ Universität Stuttgart, Pfaffenwaldring 57, 70550 Stuttgart, Germany \\ ${ }^{2}$ Institute of Nanotechnology, Karlsruhe Institute of Technology, 76344 Eggenstein-Leopoldshafen, Germany \\ ${ }^{3}$ Institute of Physics, University of Münster, 48149 Münster, Germany \\ ${ }^{4}$ Institut für Physik, Universität Rostock, Albert-Einstein-Straße 23, 18059 Rostock, Germany
}

(Received 9 November 2017; revised manuscript received 6 February 2018; published 4 May 2018)

We study the interaction of thermal rubidium atoms with the guided mode of slot waveguides integrated in a vapor cell. Slot waveguides provide strong confinement of the light field in an area that overlaps with the atomic vapor. We investigate the transmission of the atomic cladding waveguides depending on the slot width, which determines the fraction of transmitted light power interacting with the atomic vapor. An elaborate simulation method has been developed to understand the behavior of the measured spectra. This model is based on individual trajectories of the atoms and includes both line shifts and decay rates due to atom-surface interactions that we have calculated for our specific geometries using the discrete dipole approximation. Furthermore, we investigate density-dependent effects on the line widths and line shifts of the rubidium atoms in the subwavelength interaction region of a slot waveguide.

DOI: 10.1103/PhysRevX.8.021032

\section{INTRODUCTION}

The integration of atomic gases with photonic devices has the potential to miniaturize and incorporate sources, active media, and detectors on a single platform for atom-lightinteraction-based applications, such as sensing, referencing, or nonlinear optical systems. Currently, various strategies exist to interface atoms with confined light fields, for example, atoms in hollow waveguides (ARROWs) [1,2], hollow core fibers [3,4], and tapered nanofibers [5-7]. A further scheme has recently been demonstrated by combining chip-based solid core waveguides [8-10], MachZehnder interferometers [8], and ring resonators [11,12] with thermal atomic vapor. The performance of such devices depends on the efficiency of the atom-light coupling, which is conventionally improved by employing cavities to increase the interaction time. This concept has also been followed with cold atoms and nanophotonic resonators [13,14], as well as photonic crystal waveguides [15], where the interaction time is prolonged due to slow light effects. While cold atom experiments provide ideal conditions to explore the strong coupling regime, their scalability is limited because of the

\footnotetext{
*r.loew@physik.uni-stuttgart.de
}

Published by the American Physical Society under the terms of the Creative Commons Attribution 4.0 International license. Further distribution of this work must maintain attribution to the author(s) and the published article's title, journal citation, and DOI.
Subject Areas: Atomic and Molecular Physics, Photonics

large setups required to cool and trap the atoms close to dielectric structures. Although thermal vapor cells offer less precision and control, their low technical complexity and potential for miniaturization permit the realization of scalable networks. Coherent coupling between a guided mode and thermal atoms is, however, limited by the motion of the latter, such that the interaction time improvement by a cavity would only be beneficial up to a certain quality factor. Thus, many photons are required in order to observe coherent effects with conventional waveguides, as was demonstrated in Ref. [10] using pump-probe spectroscopy. A further approach to increase the coupling strength is to use slot waveguides [16-18], where the propagating mode is strongly confined between two narrow waveguide strips on a subwavelength scale. This region of high optical intensity overlaps with the atomic vapor, which enables enhanced atom-light coupling as compared to, e.g., nanofibers or solid core waveguides, where the atoms interact only with the tail of the evanescent field. The coupling strength can be characterized by $\kappa / \Gamma$, which is the ratio between the atom's spontaneous emission rate into the fundamental waveguide mode and the total decay rate determined by the environment, which can be up to 0.35 for our structures.

In this paper, we investigate the interaction between a thermal rubidium $(\mathrm{Rb})$ vapor and slot waveguides with different slot widths and lengths. Because of limitations in the fabrication process of the optical chip, the structures exhibit a gradual transition from a single ridge waveguide 
to fully opened slot waveguides. We attempt to model the intricate dynamics of atoms moving in the evanescent field of the slot mode by means of a Monte Carlo type simulation, including the specific geometry of the individual devices and the associated atom-surface interactions. The simulation results show reasonable agreement with the measurements, except for the transition region, where the slot is not yet completely developed. Additionally, we performed density-dependent measurements to study atomatom interactions within a region that is transversally restricted to a subwavelength scale defined by the penetration depth of the evanescent field.

This paper is organized as follows. In Sec. II, we describe the design of the waveguide structures and how we acquire and evaluate the transmission spectra of slot waveguides surrounded by Rb vapor. In Sec. III, we present measurements over a range of slot widths and discuss the features of the resulting spectral line shapes. Subsequently, in Sec. IV, we introduce our model based on Monte Carlo simulations and compare its outcome with the experimental findings. An important element of this model is the inclusion of atom-surface interactions, which influence the resonance frequency and lifetime of the atoms close to the waveguide. Therefore, in Sec. V, we present our methods for the calculation of Casimir-Polder potentials, taking into account the specific waveguide geometries in our experiment. Furthermore, in Sec. VI, we study the behavior of atom-atom interactions in the vicinity of a slot waveguide.

\section{WAVEGUIDE DESIGN AND DESCRIPTION OF THE EXPERIMENT}

The slot waveguides consist of two silicon nitride $\left(\mathrm{Si}_{3} \mathrm{~N}_{4}\right)$ strips separated by a gap of width $w$ (see Fig. 1). Because of the boundary conditions of Maxwell's equations, the electric field of a transverse electric (TE) mode undergoes a discontinuity at the sidewall interface of the waveguide. A separation of the two strips smaller than the evanescent decay length of the mode results in a high electric field amplitude in the slot while it is decreased in the $\mathrm{Si}_{3} \mathrm{~N}_{4}$ strips themselves. Therefore, high optical intensities can be reached in the atomic vapor region, leading to enhanced light-matter coupling. The devices are fabricated on a borosilicate substrate that is integrated into a $\mathrm{Rb}$ vapor cell, as described in Ref. [11]. We couple light in and out of the devices via grating couplers as shown in Fig. 1(a). The couplers and the Y-branch splitters are covered with an 800-nm-thick layer of silicon dioxide $\left(\mathrm{SiO}_{2}\right)$ in order to restrict the atom-light interaction only to the slot waveguide region. This is realized by using hydrogen silesquioxane (HSQ) as a resist in an additional electron beam lithography step. To protect the structures from $\mathrm{Rb}$ atoms sticking to the waveguide surface and potential chemical reactions, we additionally cover the devices with a 7-nm-thick sapphire $\left(\mathrm{Al}_{2} \mathrm{O}_{3}\right)$ coating via atomic layer deposition.
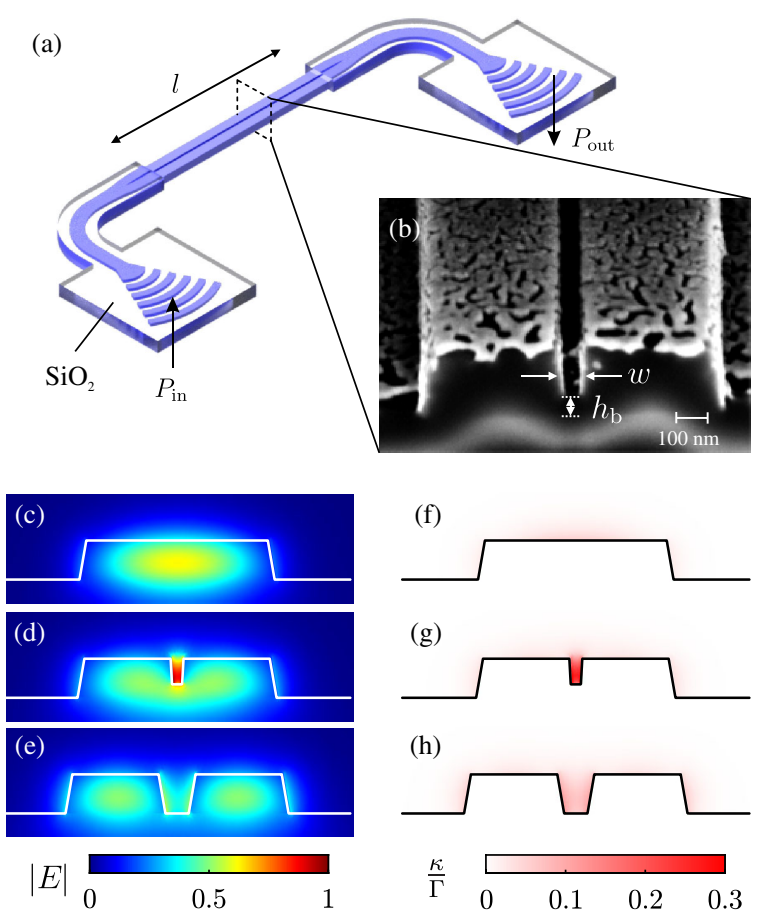

FIG. 1. Waveguide structure and mode profiles. (a) Schematic of the slot waveguides. The probe light $P_{\text {in }}$ is coupled into the waveguide via a grating coupler and guided to the slot section. The output $P_{\text {out }}$ is detected with a photo multiplier tube. (b) FIB/ SEM image of the waveguide cross section. The white patches are sputtered gold to prevent charge buildup during imaging. (c)-(e) Mode profiles of the fundamental TE mode for a closed (c), partially open (d), and fully open (e) slot waveguide. (f)-(h) Corresponding atomic decay rates $\kappa$ into the fundamental mode normalized by the total decay rate $\Gamma$.

The chip used for the presented measurements contains several slot waveguides designed to have slot widths in the range $w_{d}=30 \mathrm{~nm}-250 \mathrm{~nm}$ and uncovered domains, which are accessible for the atoms with lengths of $l=30 \mu \mathrm{m}$ and $200 \mu \mathrm{m}$. The height of the waveguides is $185 \mathrm{~nm}$, and the width of the strips is kept at $400 \mathrm{~nm}$. After the experiments have been conducted, a focused ion beam (FIB) was used to cut a trench into the structures to expose their cross sections, which have subsequently been examined by scanning electron microscopy (SEM). A sample of the images obtained with this method is shown in Fig. 1(b) for the $w_{d}=75 \mathrm{~nm}$ slot waveguide. The bright rough layer covering the surface of the sample in this micrograph is a sputtered gold layer, which forms a conducting film on the $\mathrm{Si}_{3} \mathrm{~N}_{4}$ /borosilicate sample required for the FIB/SEM treatment. Analysis of the cross sections revealed that the two waveguides with designed slot widths of $30 \mathrm{~nm}$ and $40 \mathrm{~nm}$ respectively do not actually exhibit a gap, but are solid strip waveguides instead. This is caused by the resolution limit of the used negative tone e-beam resist. Furthermore, the plasma etching process is impeded in very small openings and, therefore, the gaps of the devices with $50 \mathrm{~nm} \leq$ $w_{d} \leq 125 \mathrm{~nm}$ are not etched all the way to the substrate but 
have a remaining base of decreasing height $h_{b}$ with increasing gap size. Nevertheless, all slot waveguides with $w_{d} \geq 150 \mathrm{~nm}$ turned out to be completely developed. Additional SEM micrographs from the top, as well as under a tilted view, show very good homogeneity along the waveguides. This is further confirmed by the optical depth scaling linearly with the waveguide length when performing spectroscopy with the $l=30 \mu \mathrm{m}$ and $200 \mu \mathrm{m}$ devices.

The waveguide modes for the geometries deduced from the FIB/SEM analysis have been calculated with a finite element method using COMSOL Multiphysics. Because of the rectangular geometries of the waveguides, all modes mentioned here are TE-like and transverse magnetic (TM)like modes exhibiting a small fraction of the respective field also in the other components. For the sake of brevity, we hereafter label them as TE and TM modes. The waveguides can in principle support up to three modes; however, as discussed in the Supplemental Material [19], we believe that only the fundamental TE mode is guided in the structures.

The atomic vapor interacts with the probe light via the evanescent part of the waveguide mode, which manifests itself as an absorption signal in the transmitted light. A typical transmission spectrum, where the probe laser is scanned over the $\operatorname{Rb} D_{2}$ line with detuning $\Delta$, is shown in Fig. 2 for the $w_{d}=75 \mathrm{~nm}, l=200 \mu \mathrm{m}$ slot waveguide at an atom density of $\mathcal{N} \approx 3.5 \times 10^{14} \mathrm{~cm}^{-3}$. The motion of the atoms has a substantial effect on the line shape of the spectra. Most of them interact only for a short time with the probe light, before they leave the evanescent field, or collide with the waveguide surface, which leads to transit time broadening. In addition, the Doppler broadening is enhanced compared to free space spectroscopy because of the 1.5-1.6 times larger propagation constant. We fit the spectra with a sum of asymmetric Voigt profiles [20] corresponding to the individual dipole allowed transitions. The asymmetric profile accounts for the distribution of

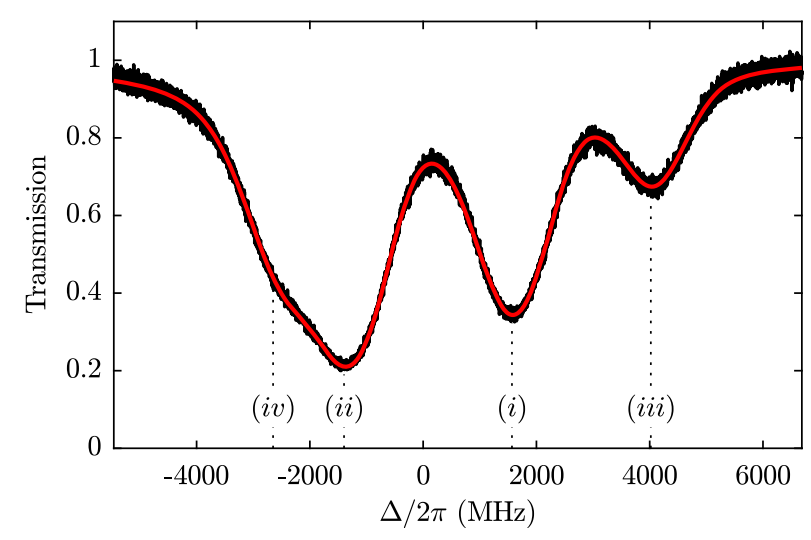

FIG. 2. Transmission spectrum of the $w_{d}=75 \mathrm{~nm}, l=200 \mu \mathrm{m}$ slot waveguide with an atom density of $\mathcal{N} \approx 3.5 \times 10^{14} \mathrm{~cm}^{-3}$. The red curve is a fit of our theory model. The dotted lines indicate the transition frequencies from the $\mathrm{Rb}^{85} S_{1 / 2} F=2$ (i), $F=3$ (ii) and $\mathrm{Rb}^{87} S_{1 / 2} F=1$ (iii), $F=2$ (iv) to the $P_{3 / 2}$ states. redshifts due to atom-surface interactions, which results in a red tail of the line shape. Figure 2 shows the excellent agreement between this model and the data.

\section{SLOT WIDTH DEPENDENCE}

The most fascinating feature of a slot waveguide is the subwavelength confinement of the light field in the low index region, which offers enhanced coupling between atoms and photons. To study the behavior of thermal atoms coupling to a slot waveguide mode, we performed spectroscopic measurements as a function of the slot width, which determines the fraction of optical power at the positions of the atoms. For each slot width, we recorded a transmission spectrum by scanning the laser over the $\mathrm{Rb} \mathrm{D}_{2}$ line, and we extracted the line shift, Voigt width, and optical depth (OD) from a fit to the spectrum, an example of which is shown in Fig. 2. Note that the signal we obtain from a slot waveguide originates from contributions of atoms in the slot itself, but also from atoms in the evanescent field of the remaining geometry [see Figs. 1(c)-1(e)]. The expected saturation intensity for the transit time broadened linewidth is reached for an atom located at the position of maximum intensity in the $w_{d}=$ $50 \mathrm{~nm}$ slot, with a mode power of approximately $15 \mathrm{nW}$. Since the 50-nm slot features the largest local intensity, we assume saturation to occur at higher powers for the other devices. In a power-dependent measurement series, we found the atoms to saturate at an input power of approximately $12 \mu \mathrm{W}$, in agreement with the estimated mode power, taking into account coupling losses and the intensity distribution of the mode. The input power for the presented experiments was $P_{\text {in }} \approx 400 \mathrm{pW}$ and, therefore, well below the power required to reach the saturation intensity. The atom density was set to $\mathcal{N} \approx 3.5 \times 10^{14} \mathrm{~cm}^{-3}$. The results of these measurements are shown as black squares in Fig. 3.

As the atoms are probed in close vicinity to the waveguide, Casimir-Polder potentials lead to a redshift of the resonance on the order of $2 \pi \times 70 \mathrm{MHz}$. A shift of $2 \pi \times 65 \mathrm{MHz}$ attributed to atoms-surface interactions was also observed for the $D_{2}$ transition of Rb probed with a ridge $\mathrm{Si}_{3} \mathrm{~N}_{4}$ waveguide [10], which is consistent with our measurement. The linewidth shown in Fig. 3(b) increases for a growing slot width up to $w_{d}=125 \mathrm{~nm}$ and drops again for larger slot widths. The rise in linewidth can be explained by a growing slot volume and, therefore, more and more atoms from inside the slot region contribute to the signal. Because these atoms have very little time to interact with the light field, they exhibit a large transit time broadened linewidth. At $w_{d}=125 \mathrm{~nm}$, the dimensions become similar to the decay length of the evanescent field of the remaining cladding and, therefore, the linewidth reaches its maximum. The further trend of the linewidth is determined by the Doppler width, which is decreasing for growing slot widths as the effective mode index declines. The OD for the $\mathrm{Rb}^{85} 5 S_{1 / 2}, F=3 \rightarrow 5 P_{3 / 2}, F=4$ transition is shown in panel (c) and is an indicator for the 

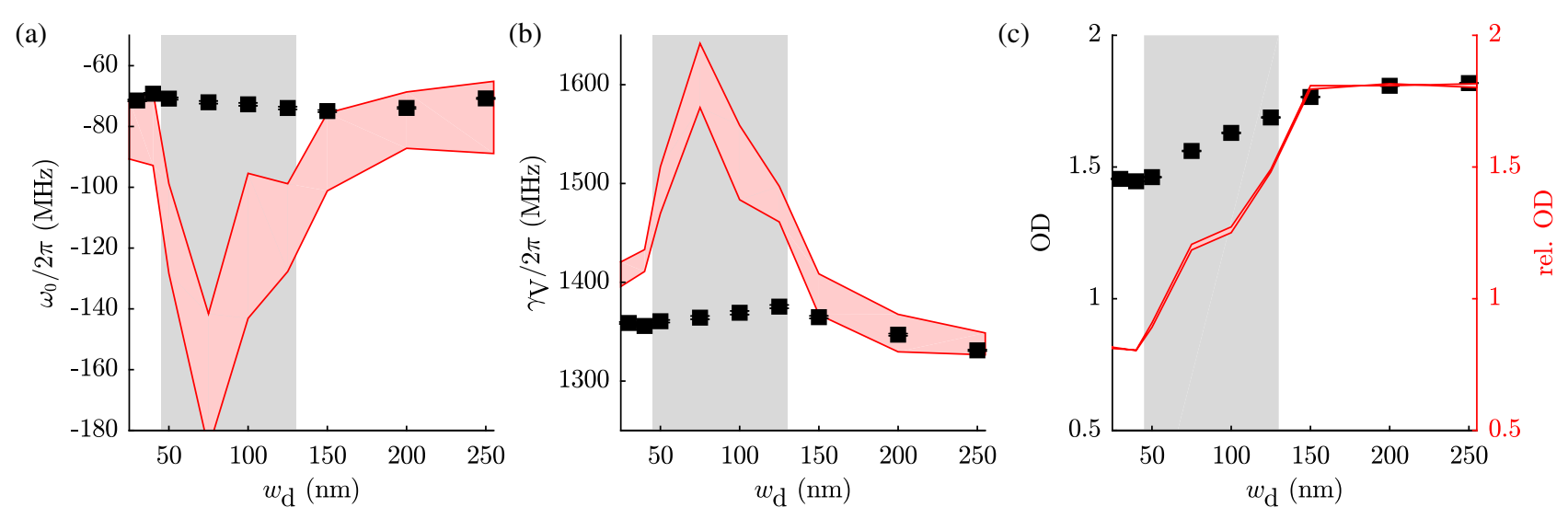

FIG. 3. Line shape parameters of the experimental spectra and Monte Carlo simulation results versus design slot width $w_{d}$. Black squares show experimental data and the red areas correspond to simulation results, taking into account the uncertainty of the $\mathrm{CP}$ potential calculations. The gray shaded area indicates the interval $50 \mathrm{~nm} \leq w_{d} \leq 125 \mathrm{~nm}$, where the slot is not yet completely developed. (a) Center frequency $\omega_{0}$ of the $D_{2}$ spectrum relative to the center frequency obtained in a reference cell. (b) Voigt width $\gamma_{V}$ (FWHM). (c) Optical depth of the $\mathrm{Rb}^{85} 5 S_{1 / 2}, F=3 \rightarrow 5 P_{3 / 2}, F=4$ transition. The simulation results for the OD are normalized to the maximum values of the measured OD and presented as relative OD (right ordinate) for a better comparison with the experimental data.

strength of the atom-light interaction (for a fixed number density and waveguide length). For atoms at rest and in the limit of low saturation, the OD is proportional to the percentage of power outside the waveguide $\eta=\iint_{r>W} d \boldsymbol{r} I(\boldsymbol{r}) / P$, where $W$ denotes the waveguide cross section, $I(\boldsymbol{r})$ is the intensity at position $\boldsymbol{r}$, and $P$ is the total power. According to our mode simulations, $\eta$ increases for growing slot widths up to its maximum at $w_{d}=125 \mathrm{~nm}$ and then slightly decreases for larger slot widths. This behavior is reproduced by the measured OD only to some degree but, as we have thermal atoms in our experiment, the OD is influenced by motional effects, and a deviation from the cold atom picture is expected, which led to the development of the model presented in the next section.

\section{MONTE CARLO SIMULATIONS}

To understand the interaction of a thermal vapor with the waveguide mode depending on the exact slot geometry, we developed a model based on Monte Carlo simulations of atomic trajectories to account for the various effects arising in this system. The simulation volume is bounded by a box around the waveguide with cross-section dimensions $w_{x} \times w_{y}$ and length $w_{z}$, where, in most cases, $w_{x}=2 \mu \mathrm{m}$, $w_{y}=1 \mu \mathrm{m}$, and $w_{z}=1 \mu \mathrm{m}$. For each atom, the timedependent optical Bloch equations of a two-level system are solved with a fourth-order Runge-Kutta method for a set of detunings $\Delta$. Optical pumping effects are negligible in this system because of the short interaction time and the low probe Rabi frequencies, thus allowing the two-level approximation. This is also obvious from Fig. 2, which does not show any indication of optical pumping.

In the initialization step, $N$ atoms are randomly placed within the simulation box and are given random velocities sampled from a Maxwell-Boltzmann distribution. Then time advances gradually and, in each time step (typically $\Delta t=1 \mathrm{ps}$ ), the position and the density matrix of every atom are recalculated. The individual Rabi frequencies $\Omega(\boldsymbol{r})$, total decay rates $\Gamma(\boldsymbol{r})$, decay rates into the fundamental mode $\kappa(\boldsymbol{r})$, and effective detunings $\Delta_{\text {eff }}\left(\boldsymbol{r}, v_{z}\right)=$ $\Delta-\Delta_{\mathrm{CP}}(\boldsymbol{r})-\beta v_{z}$, which enter the optical Bloch equations, are updated with respect to the momentary position and velocity. Here, $\Delta_{\mathrm{CP}}$ is a shift of the resonance frequency due to the Casimir-Polder $(\mathrm{CP})$ potential $U_{m}(\boldsymbol{r})$, which will be examined in detail below, and $\beta$ is the propagation constant of the waveguide mode. The waveguide geometry is implemented with the dimensions inferred from the FIBSEM measurements described earlier, and the corresponding modes are used to calculate the Rabi frequencies and decay rates into these modes. Whenever an atom collides with the waveguide surface or if it hits the transversal boundary of the simulation box, we assume that it gets deexcited and its coherences are set to zero. In the subsequent time step, the atom leaves the surface in its ground state with a new direction and speed according to Knudsen's cosine law [21]. In reality, the atom might stick to the surface for a certain time; however, adsorption and desorption rates are identical after a dynamical equilibrium has been reached for a given temperature, so it is reasonable to assume that the atom leaves the surface instantly after a collision. The experiments with different slot widths have been conducted in a density regime, where the mean free path is longer than the size of the cell, and we can, therefore, neglect collisions between atoms. For the longitudinal direction, we use periodic boundary conditions, such that an atom passes through one side of the box enters on the opposite side with the same velocity and density matrix.

We run the simulations for a certain time $T=n_{t} \times \Delta t$ (usually a few hundred nanoseconds up to microseconds) 
with $n_{t}$ time steps and discard the first few nanoseconds that are required to reach a steady state. Multiple simulations are executed with the same parameters and averaged afterwards to improve the signal-to-noise ratio. In order to compare the simulations with the experimental data, we need to determine how much light is scattered by the atoms into free space or into the waveguide mode in both the forward and backward directions. Also, we need to register the accumulated excited-state fraction of the atoms at the instance they collide with a surface or the transversal simulation boundary. We write, for the total scattered power inside the simulation volume,

$$
\begin{aligned}
P_{\mathrm{sc}}(\Delta)= & \hbar \omega_{0} \sum_{\Delta t} \sum_{i=1}^{N}\left[\left(\Gamma\left(\boldsymbol{r}_{i}, t\right)-\frac{\kappa\left(\boldsymbol{r}_{i}, t\right)}{2}\right)\right. \\
& \left.\times \frac{\sigma_{22}\left(\boldsymbol{r}_{i}, t, \Delta_{\mathrm{eff}}\right)}{n_{t}}+\frac{\sigma_{22}^{\mathrm{col}}\left(\boldsymbol{r}_{i}, t, \Delta_{\mathrm{eff}}\right)}{T}\right],
\end{aligned}
$$

where $\sigma_{22}$ denotes the population probability of an atom inside the simulation volume and $\sigma_{22}^{\text {col }}$ is the population probability of an atom that undergoes a surface collision at time step $t$. The inner sum represents an average over all atoms, and the outer sum takes care of time averaging. The first term within the square brackets accounts for the scattered power due to the decay of the excited state, which is determined by $\Gamma$. A fraction of the atoms decays into the forward propagating fundamental mode, with a rate $\kappa / 2$, and has to be subtracted from the total scattered power as this portion is added to the observed transmission signal. Excitation losses due to surface collisions are included via the second term. For $P_{\mathrm{sc}}(\Delta)$, we obtain a line shape that can be in good approximation fitted by an asymmetric Voigt profile in order to extract the line shift, line width, and amplitude of the signal, as we do for the experimental data. An example thereof is presented in Fig. 4 for the $w_{d}=$ $150 \mathrm{~nm}$ slot. To obtain the optical depth of the simulated section, we use the relation OD $=-\log \left(1-P_{\mathrm{sc}} / P_{\text {in }}\right)$.

The simulation results and the experimental data for the different slot widths are shown in Fig. 3. Since we are only interested in the relative change of the OD as a function of slot width, we normalize the ODs of the simulations to the maximum measured OD value for easier comparison to the experimental behavior. Here, the trend of increasing OD with growing slot size is reproduced by the simulation; however, the relative difference between the maximum and minimum OD is slightly larger for the simulation results. For the strip waveguides $\left(w_{d} \leq 40 \mathrm{~nm}\right)$ and the completely resolved slots $\left(w_{d} \geq 150 \mathrm{~nm}\right)$, we achieve good agreement in the line shifts and Voigt widths, even quantitatively. There are obvious discrepancies for the four slots with $50 \mathrm{~nm} \leq w_{d} \leq 125 \mathrm{~nm}$. This interval corresponds to the devices where the slot starts to form but is not yet etched all the way to the substrate.

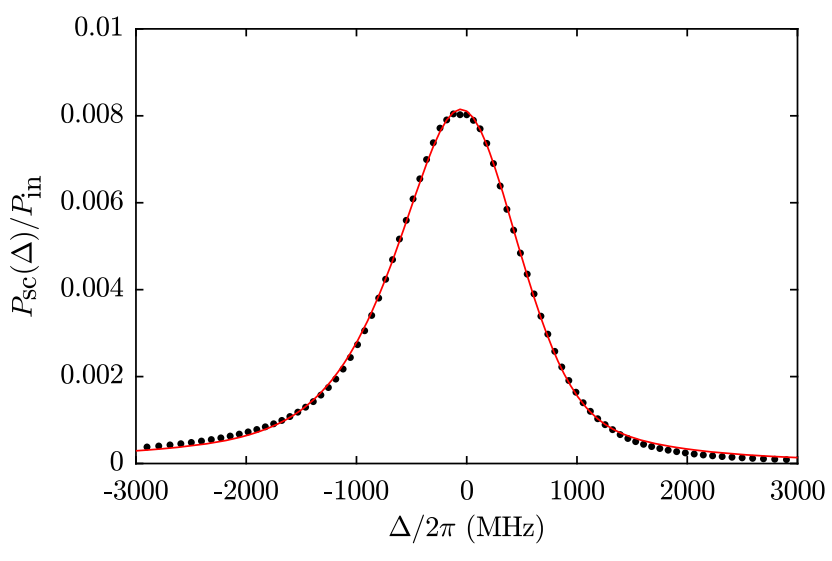

FIG. 4. Simulation result for the $w_{d}=150 \mathrm{~nm}$ slot waveguide. The black dots show the scattered power $P_{\text {sc }}$ normalized by the input power $P_{\text {in }}$ as a function of detuning $\Delta$. Also shown is the corresponding Voigt fit (red line), from which we extract the relevant line shape parameters.

We now want to discuss several possible causes for the deviations between experiments and simulations. The outliers in the line shift and linewidth within the gray shaded area of Fig. 3 suggest that the atom-light interaction might be overestimated in the slot itself. This could be caused by either a reduced atom density inside the slot in comparison to the surrounding volume or by deviations in the mode profile. We discuss the likelihood for the presence of higher order modes in the Supplemental Material [19] with the outcome that the fundamental mode should be dominant. Also, the Doppler widths extracted from fits to the experimental spectra follow the trend of the effective indices of the fundamental mode quite well, which is a further indication that the atoms interact with this mode, since the Doppler width is proportional to the effective mode index.

The mode profile could be altered by $\mathrm{Rb}$ sticking to the waveguide surface and, therefore, creating a metallic layer. In order to investigate this situation, we simulated the transmission properties of the slot waveguides with a thin metal layer on top using COMSOL. The metal layer was set up to have the optical properties of $\mathrm{Rb}$ and its thickness was adjusted such that the transmission losses were corresponding to experimentally obtained values before and after exposing the waveguides to $\mathrm{Rb}$ vapor. The result suggests a Rb layer thickness of approximately 200 pm, which, however, does not influence the mode profile perceptibly.

Another reason for less signal contribution from the slot would be a reduced atom number density within the slot as compared to the volume around the waveguide, which can arise if the adsorption rate of atoms onto the waveguide surface is much larger than the desorption rate. In this case, most of the atoms had to make it through the narrow slot opening to enter the slot region, which is restricted by the 
geometrical obstructions. Simulations with different ratios of adsorption and desorption rates showed indeed much better agreement when desorption becomes negligible compared to adsorption, but it is unclear to what extent this constructed behavior reflects the real experimental conditions.

Besides the rate, the directions of desorbing atoms also influence their interaction with the slot mode. Various experiments in surface science have reported on noncosine distributions for the directions of desorbing molecules (see, e.g., Ref. [22] and references therein). They find distributions that are strongly peaked around the surface normal and can usually be described by a $\cos ^{n} \theta$ function. Hence, we checked the influence of the angle distribution of atoms leaving the surface for several exponents $(1 \leq n \leq 100)$, but we could not obtain a better agreement between simulation and experimental results compared to the usual cosine distribution. The roughness of the waveguide surface could also lead to an anomalous adsorption and desorption behavior, not accounted for in our simulations.

The determination of the slot waveguide dimensions via SEM images of their cross sections involves further uncertainties due to possible distortions caused by electrostatic charging of the chip. Also, as one can sense from Fig. 1(b), it is difficult to pinpoint the borders of the structure in theses images. We have tested our model for a ridge waveguide with well-known dimensions and obtain very good agreement, as further described in the Supplemental Material [19]. In order to improve the quality of this model, a better knowledge of the actual waveguide geometry would be necessary, especially if the contours are rather complex, as is the case for the narrow slot waveguides. In addition, an experimental characterization of the guided modes, e.g., using 3-D near-field imaging [23], would provide valuable input data for our simulations. A simulation study of the atom-light interaction for a particular device with different stages of slot development is performed in the Supplemental Material [19].

\section{ATOM-SURFACE INTERACTIONS}

\section{A. Theoretical framework}

A major ingredient of the model presented in the previous section is the integration of atom-surface interactions. Even though atoms are neutral particles, they are electrically polarizable, for example, by groundstate fluctuations of the electromagnetic field. In the presence of macroscopic bodies, these dipole moments create mirror charges that react on the original dipole, leading to shifts of the atomic lines. We study these socalled CP interactions in the framework of macroscopic QED [24]. The key in this approach is to find the classical Green's tensor $\mathbf{G}\left(\boldsymbol{r}, \boldsymbol{r}^{\prime}, \omega\right)$ as the unique solution to the Helmholtz equation,

$$
\left[\boldsymbol{\nabla} \times \boldsymbol{\nabla} \times-\frac{\omega^{2}}{c^{2}} \epsilon(\boldsymbol{r}, \omega)\right] \mathbf{G}\left(\mathbf{r}, \boldsymbol{r}^{\prime}, \omega\right)=\boldsymbol{\delta}\left(\boldsymbol{r}-\boldsymbol{r}^{\prime}\right),
$$

with vanishing boundary conditions at infinity. Here, $\epsilon(\boldsymbol{r}, \omega)$ denotes the dielectric permittivity of the bodies. Physically, $\boldsymbol{\Gamma}_{\omega}\left(\boldsymbol{r}, \boldsymbol{r}^{\prime}\right) \equiv\left(\omega^{2} / c^{2}\right) \mathbf{G}\left(\boldsymbol{r}, \boldsymbol{r}^{\prime}, \omega\right)$ describes the electric field at point $\boldsymbol{r}$ that is created by a pointlike dipole source at $\boldsymbol{r}^{\prime}$, oscillating with angular frequency $\omega$. Thereby, the Green's tensor generalizes the static picture of mirror charges to a full electrodynamic treatment. The CP potential of an atom prepared in an eigenstate $|m\rangle$ may be given as $U_{m}(\boldsymbol{r})=U_{m}^{n r}(\boldsymbol{r})+U_{m}^{r}(\boldsymbol{r})$, with nonresonant and resonant contributions $[25,26]$,

$$
\begin{array}{r}
U_{m}^{n r}(\boldsymbol{r})=-\frac{k_{B} T}{\epsilon_{0}} \sum_{j=0}^{\infty}\left(1-\frac{1}{2} \delta_{j 0}\right) \alpha_{m}\left(i \xi_{j}\right) \operatorname{Tr} \Gamma_{i \xi_{j}}^{\mathrm{sc}}(\boldsymbol{r}, \boldsymbol{r}), \\
U_{m}^{r}(\boldsymbol{r})=\frac{1}{3 \epsilon_{0}} \sum_{k} n\left(\omega_{m k}\right)\left|\boldsymbol{d}_{m k}\right|^{2} \operatorname{Tr} \operatorname{Re} \Gamma_{\omega_{m k}}^{\mathrm{sc}}(\boldsymbol{r}, \boldsymbol{r}) .
\end{array}
$$

Here, $\xi_{j}=2 \pi k_{B} T j / \hbar$ denotes the Matsubara frequencies, $n(\omega)=\left(\exp \left[\hbar \omega /\left(k_{B} T\right)\right]-1\right)^{-1}$ is the Bose-Einstein distribution for angular frequency $\omega$ at temperature $T, \alpha_{m}(\omega)$ is the atomic polarizability, and the sum in Eq. (4) extends over all states $|k\rangle$ featuring a significant transition dipole moment $\boldsymbol{d}_{m k}=\langle m|\hat{\boldsymbol{d}}| k\rangle$. All properties of the macroscopic surroundings are encoded in $\boldsymbol{\Gamma}_{\omega}^{\mathrm{sc}}(\boldsymbol{r}, \boldsymbol{r})=\boldsymbol{\Gamma}_{\omega}(\boldsymbol{r}, \boldsymbol{r})-\boldsymbol{\Gamma}_{\omega}^{(0)}(\boldsymbol{r}, \boldsymbol{r})$, where the free-space tensor $\boldsymbol{\Gamma}_{\omega}^{(0)}(\boldsymbol{r}, \boldsymbol{r})$ is subtracted as the corresponding Lamb shift is already included in the freespace atomic spectrum.

Additionally, the imaginary part of the Green's tensor provides a measure for the local density of states (LDOS). Hence, the modified single-particle decay rate, indicating the presence of the Purcell effect [27], can be written as [24]

$\Gamma_{m}(\boldsymbol{r})=\frac{2}{3 \epsilon_{0} \hbar} \sum_{k}\left(n\left(\omega_{m k}\right)+1\right)\left|\boldsymbol{d}_{m k}\right|^{2} \operatorname{Tr} \operatorname{Im} \boldsymbol{\Gamma}_{\omega_{m k}}(\boldsymbol{r}, \boldsymbol{r})$.

For geometries with high symmetry, such as bulk media or layered planar, cylindrical, and spherical interfaces, the expressions for the Green's tensor are known analytically [28] and can be computed fast and accurately [29]. For more complex systems such as the present one, numerical techniques have to be applied.

\section{B. Potential simulations}

In order to obtain the $\mathrm{CP}$ shift $\Delta_{\mathrm{CP}}$ of the $5^{2} P_{3 / 2} \rightarrow$ $5^{2} S_{1 / 2}$ transition line in $\mathrm{Rb}$, we compute the shifts of the individual levels $5^{2} P_{3 / 2}$ and $5^{2} S_{1 / 2}$ and subtract them. The corresponding atomic polarizabilities at imaginary frequencies are given by 


$$
\alpha_{m}(i \xi)=-\frac{2}{3 \hbar} \sum_{k} \frac{\omega_{m k}\left|\boldsymbol{d}_{m k}\right|^{2}}{\omega_{m k}^{2}+\xi^{2}},
$$

where the dipole moments $\left|\boldsymbol{d}_{m k}\right|$ and energy levels were extracted from coupled cluster computations [30] and experimental data [31].

Further, the permittivities $\epsilon(\omega)$ of all involved materials need to be provided at real and imaginary frequencies. For fused silica, we utilize the data compilation by Kitamura et al. [32] and perform a numerical Kramers-Kronig transformation to obtain values at imaginary frequencies,

$$
\epsilon(i \xi)=1+\frac{2}{\pi} \int_{0}^{\infty} d \omega \frac{\omega \operatorname{Im} \epsilon(\omega)}{\omega^{2}+\xi^{2}} .
$$

For sapphire, we follow Fichet et al. [33] and use the geometric mean of the Drude-Lorentz permittivities of the two principal axes. For silicon nitrite, we utilize a DrudeLorentz model for the infrared region [34] and fitted a TaucLorentz model [35] to data in the ultraviolet regime [36]. These models are Kramers-Kronig consistent and can be evaluated at real and imaginary frequencies.

In order to compute the Green's tensors $\mathbf{G}(\boldsymbol{r}, \boldsymbol{r}, \omega)$ for the waveguide geometries, we adapt the discrete dipole approximation (DDA) [37,38]. The key idea behind DDA is to discretize only those parts of the system that differ from a background, i.e., free space or a planar substrate, with a known analytic solution. Each discretized cell acts as a polarizable pseudoparticle that scatters the field of the fluctuating atomic dipole. One can obtain an approximate result by summing up the fields reflected by each dipole (first Born approximation) or solve a large system of linear equations to obtain the exact fields, which also account for scattering among the discrete dipoles. The required equations and a review of the computational techniques required to solve them are presented in the Supplemental Material [19].

Modern DDA codes require a regular grid of equally sized dipoles, which enable a great reduction of the required memory and CPU time through a Fourier transformation approach [39-41]. Such techniques are mandatory for systems as large as ours, as otherwise, in a system with $N=10^{5}$ dipoles, the interaction matrices would consume $\left(3 \times 10^{5}\right)^{2} \times 16$ bytes $=1.44 \mathrm{~TB}$ of memory. However, these regular grids also lead to computational limitations when atoms come very close to the surface, where very small unit cells are required. In these cases, the overall computational domain can be truncated as at imaginary frequencies only those parts of the bodies close to the atom contribute significantly. By contrast, the scattering effects at real frequencies always require a computational domain on the order of the wavelength, which leads to a substantial increase in the computational effort. Therefore, we use the full DDA approach only at imaginary frequencies and invoke a first Born
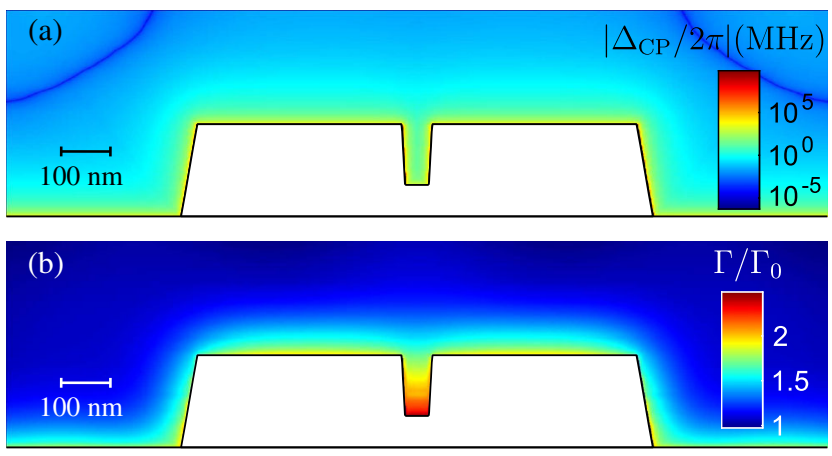

FIG. 5. Casimir-Polder line shift (a) and modified decay rate (b) plotted in the cross section of a waveguide with $w_{d}=75 \mathrm{~nm}$. Note that, in (a), the color scale corresponds to the logarithm of the absolute value of the shift. In the vicinity of the surface, the transition frequency is redshifted. However, at larger distances, a positive resonant potential contribution cancels (dark blue region) and overcomes the negative off-resonant potential such that the total shift becomes positive and the line is blueshifted. Further, the total decay rate $\Gamma$ of the $5^{2} P_{3 / 2}$ state (b) approaches its vacuum value of $\Gamma_{0}$ at large distances.

approximation at real frequencies where an adjustable grid can be used. Here, this approximation introduces an uncertainty of up to $20 \%$ to the predicted potential shift and of up to $30 \%$ to the predicted decay rate. An exemplary result of the modified decay rate and the Casimir-Polder induced shift of the transition frequency obtained by this approach is displayed in Fig. 5.

To obtain the fraction of the spontaneous emission that is reemitted into the waveguide mode, the Green's tensor in Eq. (5) can be expanded into a corresponding basis [42]. In a waveguide with low losses, the emission rate of a randomly oriented dipole into mode $\mu$ takes the form [43]

$$
\Gamma_{\mu}(\boldsymbol{r})=\frac{\pi c^{3} \epsilon_{0}\left|\boldsymbol{E}_{\mu}(\boldsymbol{r})\right|^{2}}{2 \omega^{2} P_{\mu}} \Gamma_{0}
$$

where $\Gamma_{0}$ denotes the vacuum decay rate, $\boldsymbol{E}_{\mu}$ the field distribution of the mode, and $P_{\mu}=\frac{1}{2} \int\left(\boldsymbol{E}_{\mu} \times \boldsymbol{H}_{\mu}^{*}\right) \cdot d \boldsymbol{A}$ the corresponding power. The decay rate into the fundamental mode is denoted by $\kappa(\boldsymbol{r})=\Gamma_{\mathrm{TEO}}(\boldsymbol{r})$ and is shown in Figs. 1(f)-1(h) for different slot widths. Inside the narrow slots, the value of $\kappa$ can become considerably large. For the $w_{d}=50 \mathrm{~nm}$ waveguide, we calculate $\kappa=0.35$.

\section{DENSITY-DEPENDENT MEASUREMENTS}

Finally, we studied atom-atom interactions in the evanescent field of a slot waveguide. Therefore, we recorded number-density-dependent transmission spectra with the $w_{d}=50 \mathrm{~nm}, l=30 \mu \mathrm{m}$, and $l=200 \mu \mathrm{m}$ devices. The line center $\omega_{0}$ and Voigt width (FWHM) are extracted from fits to the spectra as described earlier. The Lorentzian contribution to the Voigt width is calculated by assuming a 




FIG. 6. Density-dependent measurements in the 50-nm slot waveguides. (a) Lorentzian linewidth for the $l=30 \mu \mathrm{m}$ (blue circles) and $l=200 \mu \mathrm{m}$ (green triangles) devices. (b) Corresponding line shift with linear fit.

Doppler broadening corresponding to the cell temperature and the effective mode index of the waveguide. The result of these measurements is shown in Fig. 6. The density dependence of the Lorentzian width is plotted in part (a) and shows a nearly linear increase with the growing number density. This behavior is attributed to a self-broadening contribution to the homogeneous linewidth due to resonant dipole-dipole interactions $[44,45]$. Assuming this, the total Lorentzian width is given by $\Gamma_{\text {tot }}=\Gamma+\Gamma_{\mathrm{TT}}+\beta \mathcal{N}$, where $\Gamma_{\mathrm{TT}}$ is the transit time broadening, $\beta$ is the self-broadening coefficient, and $\mathcal{N}$ is the number density. By fitting a linear function to the data, we find for the self-broadening coefficient $\beta / 2 \pi=(1.5 \pm 0.3) \times 10^{-7} \mathrm{Hzcm}^{3}$, which agrees within uncertainties with the $\mathrm{Rb} D_{2}$ self-broadening coefficient $\beta / 2 \pi=(1.1 \pm 0.17) \times 10^{-7} \mathrm{Hzcm}^{3}$ determined via attenuated selective reflection spectroscopy [46].

With increasing number density, the line shifts linearly to lower frequencies as shown in Fig. 6(b). The gradient of the line shift is determined from a linear fit to be $\Delta_{\text {tot }} / \mathcal{N}=2 \pi \times(-0.25 \pm 0.07) \times 10^{-7} \mathrm{Hzcm}^{3}$. Two mechanisms are known to contribute to a density-dependent shift in a thermal vapor: a collision induced shift $\Delta_{\text {col }}$ due to transfer of excitation between an excited and a ground-state atom [47] and a shift induced by dipole-dipole interactions $\Delta_{\text {dd }}$ between the atoms [48]. Further, the total line shift $\Delta_{\text {tot }}$ contains an offset for $\mathcal{N} \rightarrow 0$ attributed to the atom-surface interactions $\Delta_{\mathrm{CP}}$ discussed in the previous section; hence, $\Delta_{\text {tot }}=\Delta_{\mathrm{CP}}+\Delta_{\text {col }}+\Delta_{\mathrm{dd}}$. The gradient of our line shift is consistent with the collisional shift of $\Delta_{\text {col }} / \mathcal{N}=2 \pi \times$ $(-0.25 \pm 0.01) \times 10^{-7} \mathrm{~Hz} \mathrm{~cm}^{3}$ for the $\mathrm{Rb} D_{2}$ line measured by Keaveney et al. [48], assuming that the dipoledipole contributions are negligible. A detailed study of the latter would require a (DDA) simulation of the scattering of the guided mode by the atomic vapor, which accounts for the dipole-dipole correlations and inhomogeneous (Doppler) broadening of the atoms [49,50]. Further, all dipole-dipole interactions taking place in the vicinity of the waveguide surface would have to incorporate the macroscopic environment requiring an additional scattering simulation for each pair of atoms. The implementation of this complex scheme is left to future work.

\section{CONCLUSION AND OUTLOOK}

In conclusion, we have studied the transmission of slot waveguides immersed in $\mathrm{Rb}$ vapor for different waveguide geometries. For the limiting cases of a single strip waveguide and a fully developed slot waveguide, our model adequately reproduces the line shape parameters of the measured transmission spectra. The calculation of the $\mathrm{CP}$ potentials for the actual geometries and material compositions allowed us to obtain quantitative agreement between the simulated and measured line shifts in the aforementioned cases. We have discussed several reasons for the deviations between experiments and simulations; however, further systematic investigations need to be done in order to clarify some uncertainties in the presented system. Therefore, we intend to fabricate samples with varying etching depth to study the influence of the remaining pedestal in the narrow slots. Moreover, we plan to cover the top and side domains of the waveguides, e.g., with an $\mathrm{SiO}_{2}$ mask and etch the substrate underneath the slot, to obtain a signal originating solely from atoms inside the slot. The suggested changes of the waveguide design and improved fabrication methods should allow us to explore the enhanced atom-light coupling capabilities of slot waveguides in upcoming experiments. Bending the slot waveguide into a ring would combine the benefits of a small transversal mode volume in the slot and multiple round trips of photons inside the ring resonator in order to further enhance the coupling of atoms to a photonic mode [51].

Furthermore, we studied density-dependent line shifts and broadenings of the waveguide transmission spectra for a slot width of $50 \mathrm{~nm}$. The observed increase in linewidth can be attributed to self-broadening, whereas the line shift can be explained with a collisional shift. However, a deeper understanding of the underlying mechanisms for the line shift in our particular situation is required and will be a subject of future research.

The role of resonant dipole-dipole interactions between atoms is a topic of current debate [52,53], which indicates 
the need for improvements of the existing theories. An indepth understanding of this subject is not only relevant for a microscopic description of fundamental phenomena, such as light propagation in a medium, but also for atom-based applications like atomic clocks [54]. Because of the subwavelength confinement of the light field, atoms inside a slot waveguide might offer new ways to study dipoledipole interactions in a quasi-one-dimensional arrangement. Reducing the dimensionality of the system, as recently realized for two dimensions [48,53], could provide new insights into this field of research.

\section{ACKNOWLEDGMENTS}

We gratefully acknowledge illuminating discussions with Dr. M. Yurkin on the DDA approach. We acknowledge support by the Deutsche Forschungsgemeinschaft (DFG) with the Projects No. LO1657/2 and No. SCHE612/2, and the Alliance for Quantum Innovation Program of the Center for Integrated Quantum Science and Technologies (IQST) at the University of Stuttgart. N. G. acknowledges support by the Karlsruhe School of Optics and Photonics (KSOP). H. K. acknowledges support from the Carl-Zeiss-Foundation.

[1] W. Yang, D. B. Conkey, B. Wu, D. Yin, A. R. Hawkins, and H. Schmidt, Atomic Spectroscopy on a Chip, Nat. Photonics 1, 331 (2007).

[2] H. Schmidt and A. R. Hawkins, Atomic Spectroscopy and Quantum Optics in Hollow-Core Waveguides, Laser Photonics Rev. 4, 720 (2010).

[3] G. Epple, K. S. Kleinbach, T. G. Euser, N. Y. Joly, T. Pfau, P. St. J. Russell, and R. Löw, Rydberg Atoms in Hollow-Core Photonic Crystal Fibres, Nat. Commun. 5, 4132 (2014).

[4] A. D. Slepkov, A. R. Bhagwat, V. Venkataraman, P. Londero, and A. L. Gaeta, Spectroscopy of Rb Atoms in Hollow-Core Fibers, Phys. Rev. A 81, 053825 (2010).

[5] R. Garcia-Fernandez, W. Alt, F. Bruse, C. Dan, K. Karapetyan, O. Rehband, A. Stiebeiner, U. Wiedemann, D. Meschede, and A. Rauschenbeutel, Optical Nanofibers and Spectroscopy, Appl. Phys. B 105, 3 (2011).

[6] S. M. Hendrickson, M. M. Lai, T. B. Pittman, and J. D. Franson, Observation of Two-Photon Absorption at Low Power Levels Using Tapered Optical Fibers in Rubidium Vapor, Phys. Rev. Lett. 105, 173602 (2010).

[7] S. M. Spillane, G. S. Pati, K. Salit, M. Hall, P. Kumar, R. G. Beausoleil, and M. S. Shahriar, Observation of Nonlinear Optical Interactions of Ultralow Levels of Light in a Tapered Optical Nanofiber Embedded in a Hot Rubidium Vapor, Phys. Rev. Lett. 100, 233602 (2008).

[8] R. Ritter, N. Gruhler, W. H. P. Pernice, H. Kübler, T. Pfau, and R. Löw, Atomic Vapor Spectroscopy in Integrated Photonic Structures, Appl. Phys. Lett. 107, 041101 (2015).

[9] L. Stern, B. Desiatov, I. Goykhman, and U. Levy, Nanoscale Light-Matter Interactions in Atomic Cladding Waveguides, Nat. Commun. 4, 1548 (2013).

[10] L. Stern, B. Desiatov, N. Mazurski, and U. Levy, Strong Coupling and High-Contrast All-Optical Modulation in
Atomic Cladding Waveguides, Nat. Commun. 8, 14461 (2017).

[11] R. Ritter, N. Gruhler, W. H. P. Pernice, H. Kübler, T. Pfau, and R. Löw, Coupling Thermal Atomic Vapor to an Integrated Ring Resonator, New J. Phys. 18, 103031 (2016).

[12] L. Stern, R. Zektzer, N. Mazurski, and U. Levy, Enhanced Light-Vapor Interactions and All Optical Switching in a Chip Scale Micro-ring Resonator Coupled with Atomic Vapor, Laser Photonics Rev. 10, 1016 (2016).

[13] T. Aoki, B. Dayan, E. Wilcut, W. P. Bowen, A. S. Parkins, T. J. Kippenberg, K. J. Vahala, and H. J. Kimble, Observation of Strong Coupling Between One Atom and a Monolithic Microresonator, Nature (London) 443, 671 (2006).

[14] T. G. Tiecke, J. D. Thompson, N. P. de Leon, L. R. Liu, V. Vuletic, and M. D. Lukin, Nanophotonic Quantum Phase Switch with a Single Atom, Nature (London) 508, 241 (2014).

[15] A. Goban et al., Atom-Light Interactions in Photonic Crystals, Nat. Commun. 5, 3808 (2014).

[16] V. R. Almeida, Q. Xu, C. A. Barrios, and M. Lipson, Guiding and Confining Light in Void Nanostructure, Opt. Lett. 29, 1209 (2004).

[17] Y. C. Jun, R. M. Briggs, H. A. Atwater, and M. L. Brongersma, Broadband Enhancement of Light Emission in Silicon Slot Waveguides, Opt. Express 17, 7479 (2009).

[18] Q. Quan, I. Bulu, and M. Lončar, Broadband Waveguide QED System on a Chip, Phys. Rev. A 80, 011810 (2009).

[19] See Supplemental Material at http://link.aps.org/ supplemental/10.1103/PhysRevX.8.021032 for a discussion on higher order modes and further information on the simulation model.

[20] M. Schmid, H.-P. Steinrück, and J. M. Gottfried, A New Asymmetric Pseudo-Voigt Function for More Efficient Fitting of XPS Lines, Surf. Interface Anal. 46, 505 (2014).

[21] M. Knudsen, Das Cosinusgesetz in der Kinetischen Gastheorie, Ann. Phys. (Paris) 353, 1113 (1916).

[22] G. Comsa and R. David, Dynamical Parameters of Desorbing Molecules, Surf. Sci. Rep. 5, 145 (1985).

[23] J. I. Ziegler, M. W. Pruessner, B. S. Simpkins, D. A. Kozak, D. Park, F. K. Fatemi, and T. H. Stievater, 3-D Near-Field Imaging of Guided Modes in Nanophotonic Waveguides, Nanophotonics 6, 187 (2017).

[24] S. Scheel and S. Y. Buhmann, Macroscopic QED-Concepts and Applications, Acta Phys. Slovaca 58, 675 (2008).

[25] S. Y. Buhmann and S. Scheel, Thermal Casimir versus Casimir-Polder Forces: Equilibrium and Nonequilibrium Forces, Phys. Rev. Lett. 100, 253201 (2008).

[26] S. Y. Buhmann, Dispersion Forces II (Springer, Berlin Heidelberg, 2012).

[27] E. M. Purcell, Spontaneous Emission Probabilities at Radio Frequencies, in Confined Electrons and Photons (Springer, New York, 1995), p. 839.

[28] W. C. Chew, Wave and Fields in Inhomogeneous Media (IEEE Press, New York, 1994).

[29] M. Paulus, P. Gay-Balmaz, and O. J. F. Martin, Accurate and Efficient Computation of the Green's Tensor for Stratified Media, Phys. Rev. E 62, 5797 (2000).

[30] M. S. Safronova and U. I. Safronova, Critically Evaluated Theoretical Energies, Lifetimes, Hyperfine Constants, and Multipole Polarizabilities in Rb 87, Phys. Rev. A 83, 052508 (2011). 
[31] J. E. Sansonetti, Wavelengths, Transition Probabilities, and Energy Levels for the Spectra of Rubidium (Rb I through $R b$ XXXVII), J. Phys. Chem. Ref. Data 35, 301 (2006).

[32] R. Kitamura, L. Pilon, and M. Jonasz, Optical Constants of Silica Glass from Extreme Ultraviolet to Far Infrared at near Room Temperature, Appl. Opt. 46, 8118 (2007).

[33] M. Fichet, F. Schuller, D. Bloch, and M. Ducloy, Van der Waals Interactions between Excited-State Atoms and Dispersive Dielectric Surfaces, Phys. Rev. A 51, 1553 (1995).

[34] T. S. Eriksson and C. G. Granqvist, Infrared Optical Properties of Silicon Oxynitride Films: Experimental Data and Theoretical Interpretation, J. Appl. Phys. 60, 2081 (1986).

[35] G. E. Jellison, Jr. and F. A. Modine, Parameterization of the Optical Functions of Amorphous Materials in the Interband Region, Appl. Phys. Lett. 69, 371 (1996).

[36] E. D. Palik, Handbook of Optical Constants of Solids II (Academic Press, New York, 1991).

[37] M. Paulus and O.J. F. Martin, Light Propagation and Scattering in Stratified Media: A Green's Tensor Approach, J. Opt. Soc. Am. A 18, 854 (2001).

[38] M. A. Yurkin and A. G. Hoekstra, The Discrete Dipole Approximation: An Overview and Recent Developments, J. Quant. Spectrosc. Radiat. Transfer 106, 558 (2007).

[39] P. J. Flatau, B. T. Draine, and G. L. Stephens, Light Scattering by Rectangular Solids in the Discrete-Dipole Approximation: A New Algorithm Exploiting the Block-Toeplitz Structure, J. Opt. Soc. Am. A 7, 593 (1990).

[40] R. Schmehl, B. M. Nebeker, and E. Dan Hirleman, DiscreteDipole Approximation for Scattering by Features on Surfaces by Means of a Two-Dimensional Fast Fourier Transform Technique, J. Opt. Soc. Am. A 14, 3026 (1997).

[41] B. E. Barrowes, F. L. Teixeira, and J. A. Kong, Fast Algorithm for Matrix-Vector Multiply of Asymmetric Multilevel Block-Toeplitz Matrices in 3-D Scattering, Microwave Opt. Technol. Lett. 31, 28 (2001).

[42] T. Søndergaard and B. Tromborg, General Theory for Spontaneous Emission in Active Dielectric Microstructures: Example of a Fiber Amplifier, Phys. Rev. A 64, 033812 (2001).

[43] Y. Chen, T. R. Nielsen, N. Gregersen, P. Lodahl, and J. Mørk, Finite-Element Modeling of Spontaneous Emission of a Quantum Emitter at Nanoscale Proximity to Plasmonic Waveguides, Phys. Rev. B 81, 125431 (2010).

[44] E. L. Lewis, Collisional Relaxation of Atomic Excited States, Line Broadening and Interatomic Interactions, Phys. Rep. 58, 1 (1980).

[45] L. Weller, R. J. Bettles, P. Siddons, C. S. Adams, and I. G. Hughes, Absolute Absorption on the Rubidium D1 Line Including Resonant Dipole-Dipole Interactions, J. Phys. B 44, 195006 (2011).

[46] R. Kondo, S. Tojo, T. Fujimoto, and M. Hasuo, Shift and Broadening in Attenuated Total Reflection Spectra of the Hyperfine-Structure-Resolved $\mathrm{d}_{2}$ Line of Dense Rubidium Vapor, Phys. Rev. A 73, 062504 (2006).

[47] J. Cooper and D. N. Stacey, Effect of Transfer of Excitation on Resonance Broadening, Phys. Lett. A 46, 299 (1973).

[48] J. Keaveney, A. Sargsyan, U. Krohn, I. G. Hughes, D. Sarkisyan, and C. S. Adams, Cooperative Lamb Shift in an Atomic Vapor Layer of Nanometer Thickness, Phys. Rev. Lett. 108, 173601 (2012).

[49] J. Pellegrino, R. Bourgain, S. Jennewein, Y. R. P. Sortais, A. Browaeys, S. D. Jenkins, and J. Ruostekoski, Observation of Suppression of Light Scattering Induced by DipoleDipole Interactions in a Cold-Atom Ensemble, Phys. Rev. Lett. 113, 133602 (2014).

[50] S. D. Jenkins, J. Ruostekoski, J. Javanainen, R. Bourgain, S. Jennewein, Y.R.P. Sortais, and A. Browaeys, Optical Resonance Shifts in the Fluorescence of Thermal and Cold Atomic Gases, Phys. Rev. Lett. 116, 183601 (2016).

[51] N. Rotenberg, P. Türschmann, H. R. Haakh, D. MartinCano, S. Götzinger, and V. Sandoghdar, Small Slot Waveguide Rings for On-Chip Quantum Optical Circuits, Opt. Express 25, 5397 (2017).

[52] J. Javanainen, J. Ruostekoski, Y. Li, and S.-M. Yoo, Exact Electrodynamics versus Standard Optics for a Slab of Cold Dense Gas, Phys. Rev. A 96, 033835 (2017).

[53] T. Peyrot, Y. R. P. Sortais, A. Browaeys, A. Sargsyan, D. Sarkisyan, J. Keaveney, I. G. Hughes, and C. S. Adams, The Collective Lamb Shift of a Nanoscale Atomic Vapour Layer within a Sapphire Cavity, arXiv:1801.01773.

[54] D. E. Chang, J. Ye, and M. D. Lukin, Controlling DipoleDipole Frequency Shifts in a Lattice-Based Optical Atomic Clock, Phys. Rev. A 69, 023810 (2004). 